\title{
Editorial
}
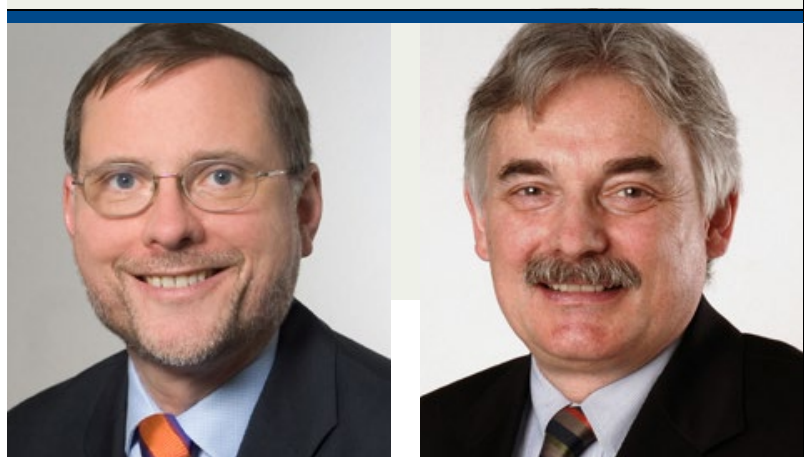

"Wir freuen uns, Ihnen auch in den nächsten Jahren eine Tagung der besonderen Art anbieten zu können."

\section{Derma Update: eine 10-jährige Erfolgsgeschichte}

m Jahr 2006 hat uns die Firma „med update“ den Vorschlag unterbreitet, in jährlichen Abständen eine neue Fortbildungsveranstaltung - Derma Update - zu organisieren. Zu diesem Zeitpunkt waren bereits einige Update-Veranstaltungen anderer Fachrichtungen mit Erfolg ins Leben gerufen worden. Vor dem Hintergrund der schon damals dicht gedrängten dermatologischen Fortbildungslandschaft erschien uns die Initiierung einer weiteren Veranstaltungsreihe eine enorme Herausforderung zu sein. Wir haben Pro und Kontra ausführlich am Rande eines Photobiologie-Kongresses in Chile bei einer Autofahrt von Santiago nach Valparaíso erwogen und haben dadurch herzlich wenig von der an uns vorbeiziehenden schönen Landschaft mitbekommen. Das attraktive Konzept, zu jedem Thema eine Auswahl der neuesten Literatur der letzten beiden Jahre zu präsentieren und gleichzeitig kritisch zu bewerten, sowie den Teilnehmern ein ausführliches mit vielen neuen Zitaten versehenes Manuskript zu übergeben, überzeugte uns letzten Endes.

Die Dermatologie ist ein sehr komplexes Fach und dementsprechend war es eine Herausforderung, die richtigen Themen zu wählen. Es erschien sinnvoll, besonders wichtige und im Hinblick auf Pathogenese und neue Therapien äußerst aktuelle Themen wie Psoriasis, atopisches Ekzem, Geno- und Autoimmundermatosen sowie Melanom jährlich zu wiederholen. Andere, in Bezug auf rezente Publikationen etwas weniger aktuelle Themen sollten als „Hot Topics“ alle zwei bis drei Jahre präsentiert werden.

Das erste Derma Update fand 2007 in Wiesbaden im Hotel Dorint statt und war mit 336 Teilnehmern schon sehr gut besucht und wie den Reaktionen der Teilnehmer eindeutig zu entnehmen war bereits ein großer Erfolg. Seit damals melden sich viele begeisterte Zuhörer noch vor Ort für das nächste Derma Update an. Im Jahr danach war der Saal im Hotel Dorint bereits zu klein und viele Interessierte bekamen keinen Platz mehr. Demnach wechselten wir ab 2009 in das Kurhaus und haben seither mit nicht ganz 1.000 Teilnehmern das Limit dieses schönen Kongresshauses erreicht. Noch immer mussten wir viele Kolleginnen und Kollegen, die mittlerweile in großer Zahl auch aus der Schweiz und Österreich anreisten, auf das kommende Jahr vertrösten. Wir entschlossen uns daher 2010 für Berlin als zweiten Veranstaltungsort. Mit nunmehr 1.800 Teilnehmern sind wir an der Grenze unsere Möglichkeiten angelangt und müssen erwägen, ob eine nochmalige Erweiterung sinnvoll und den Referenten zumutbar ist.

Es ist uns ein besonderes Anliegen, den Referenten für ihre hervorragenden Beiträge, den enormen Zeitaufwand und ihre Disziplin unseren tiefsten Dank auszusprechen. Unser Dank gilt auch dem engagierten Team von Med Update, ohne deren steten Einsatz eine derartige Veranstaltung nicht zu bewältigen wäre. Ihrem Engagement verdanken wir auch unser seit 2013 bestehendes soziales Projekt zur Unterstützung des Aufbaus einer dermatologischen Abteilung in Kambodscha. Der Spendenaufruf wurde von den Teilnehmern großzügig angenommen und wir konnten der Klinik in Phnom Penh jedes Jahr eine erhebliche Summe zur Verfügung stellen. Auch Abbvie sei an dieser Stelle gedankt für die von Anfang an bestehende finanzielle Unterstützung, ohne die Programmgestaltung in irgendeiner Weise zu beeinflussen.

Für alle, die bislang keine Gelegenheit hatten, ein Derma Update zu besuchen, haben wir in dieser Sonderausgabe von hautnah dermatologie fünf Vorträge ausgesucht, die die inhaltliche Vielfalt der Veranstaltungen repräsentieren: Von der Melanomnachsorge über das Management der Psoriasis bis zur Polyarteriitis nodosa. Wir hoffen, Sie für das Konzept begeistern zu können und freuen uns, Ihnen auch in den nächsten Jahren mit unserem bewährten Team eine Tagung der besonderen Art anbieten zu können.

Mit herzlichen Grüßen

Thomas Schwarz \& Thomas Luger 